\author{
Romain Seil ${ }^{1,2}$. Caroline Mouton' $\cdot$ Philipp Schuster ${ }^{3,4}$ - Vasileios A. Raoulis ${ }^{5}$. \\ Alexander Hoffmann ${ }^{1}$. Dietrich Pape ${ }^{1}$ - Mathieu Ollivier ${ }^{6,7}$ \\ 'Department of Orthopaedic Surgery, Centre Hospitalier de Luxembourg-Clinique d'Eich, Luxemburg, \\ Luxemburg \\ ${ }^{2}$ Sports Medicine Research Laboratory, Public Research Centre for Health, Luxemburg, Luxemburg \\ ${ }^{3}$ Departement of Arthroscopy and Sports Medicine, Orthopedic Hospital Markgröningen, Markgröningen, \\ Deutschland \\ ${ }^{4}$ Clinic Nuremberg, Department of Orthopedics and Traumatology, Paracelsus Medical Private University, \\ Nürnberg, Deutschland \\ ${ }^{5}$ Department of Orthopaedic Surgery, Faculty of Medicine, School of Health Sciences, University of \\ Thessalia, Larissa, Griechenland \\ ${ }^{6}$ Department of Orthopedics and Traumatology, Institute of Movement and Locomotion, St Marguerite \\ Hospital, Marseille, Frankreich \\ ${ }^{7}$ Department of Orthopedics and Traumatology, Institute for Locomotion, APHM, CNRS, ISM, Sainte- \\ Marguerite Hospital, Aix Marseille University, Marseille, Frankreich
}

\title{
Lösungen für häufige Komplikationen bei Beinachsenkorrekturen
}

Eine der Hauptursachen, weshalb kniegelenknahe Umstellungsosteotomien (USO) in den 1990er und 2000er Jahren sehr stark an Beliebtheit verloren hatten, war ihre hohe Komplikationsrate. In neueren Publikationen konnte die Zahl klinisch relevanter Komplikationen um oder unter die 5\%-Marke gesenkt werden (•Tab. 1), was für Eingriffe dieses Schweregrads akzeptabel erscheint. Seit der Entwicklung von Plattenfixateuren zeichnen Beinachsenkorrekturen sich durch eine hohe Primärstabilität, einen geringen Korrekturverlust und eine schnellere Nachbehandlung aus, als dies vor der Jahrtausendwende der Fall war [34]. Bei guter Indikationsstellung, adäquater präoperativer Planung und technisch korrekter Durchführung hat die hohe Zuverlässigkeit der Methode dazu geführt, dass die Indikationsstellungen erweitert und verfeinert sowie auch die Voraussetzungen für eventuelle Konversionen zur Endoprothetik wesentlich verbessert werden konnten.

Die vorliegende Arbeit bezieht sich ausschließlich auf Beinachsenkorrekturen mit Osteosyntheseplatten in der Frontalebene. Die selteneren Derotations-, Flexions- oder Extensionsosteotomien
Tab. 1 Komplikationsraten vor der Ära der Plattenfixateure mit neueren Arbeiten (ohne Anspruch auf Vollständigkeit)

\begin{tabular}{|c|c|c|c|c|}
\hline Autor & $\begin{array}{l}\text { Jahr der } \\
\text { Publikation }\end{array}$ & $\begin{array}{l}\text { Art der Umstellungsosteoto- } \\
\text { mie }\end{array}$ & $\begin{array}{l}\text { Patien- } \\
\text { tenzahl }\end{array}$ & $\begin{array}{l}\text { Komplika- } \\
\text { tionsrate (\%) }\end{array}$ \\
\hline \multicolumn{5}{|c|}{ Frühe Operationstechniken } \\
\hline Song [36] & 2010 & $\begin{array}{l}\text { PTO, lateral schließend (Klam- } \\
\text { mern) } \\
\text { PTO, medial öffnend (kein Plat- } \\
\text { tenfixateur) }\end{array}$ & 194 & 24 \\
\hline \multirow{2}{*}{$\begin{array}{l}\text { Van den } \\
\text { Bekerom [41] }\end{array}$} & 2008 & - & 40 & 62,5 \\
\hline & 2010 & - & 138 & 19 \\
\hline \multicolumn{5}{|c|}{ Aktuelle Operationstechniken (Plattenfixateure) } \\
\hline Flörkemeier [9] & 2013 & PTO, medial öffnend & 533 & 6 \\
\hline Palmer [25] & 2018 & PTO, medial öffnend & 528 & 3 \\
\hline Palmer [25] & 2018 & DFO, medial schließend & 108 & 4 \\
\hline Niemeyer [24] & 2008 & PTO, medial öffnend & 46 & 4,3 \\
\hline
\end{tabular}

Tab. 2 Bekannten Komplikationen nach kniegelenknahen Osteotomien

\begin{tabular}{|c|c|c|}
\hline Unterkorrektur & Instabilität & Fibulapseudarthrose \\
\hline Überkorrektur & $\begin{array}{l}\text { Beinlängen- } \\
\text { veränderung }\end{array}$ & Patellofemorales Schmerzsyndrom \\
\hline Fraktur der Gegenkortikalis & Streckdefizit & Infektionen \\
\hline $\begin{array}{l}\text { Pseudarthrose/verzögerte } \\
\text { Knochenheilung }\end{array}$ & $\begin{array}{l}\text { Genu recurva- } \\
\text { tum }\end{array}$ & $\begin{array}{l}\text { Gefäßkomplikationen (Beinvenenthrombose, } \\
\text { arterielle Schädigung) }\end{array}$ \\
\hline Implantatversagen & $\begin{array}{l}\text { Tibiaplateau- } \\
\text { fraktur }\end{array}$ & Prätibiale Schwellung \\
\hline Läsion des N. peronaeus & $\begin{array}{l}\text { Rotations- } \\
\text { fehlstellung }\end{array}$ & Kompartmentsyndrom \\
\hline
\end{tabular}




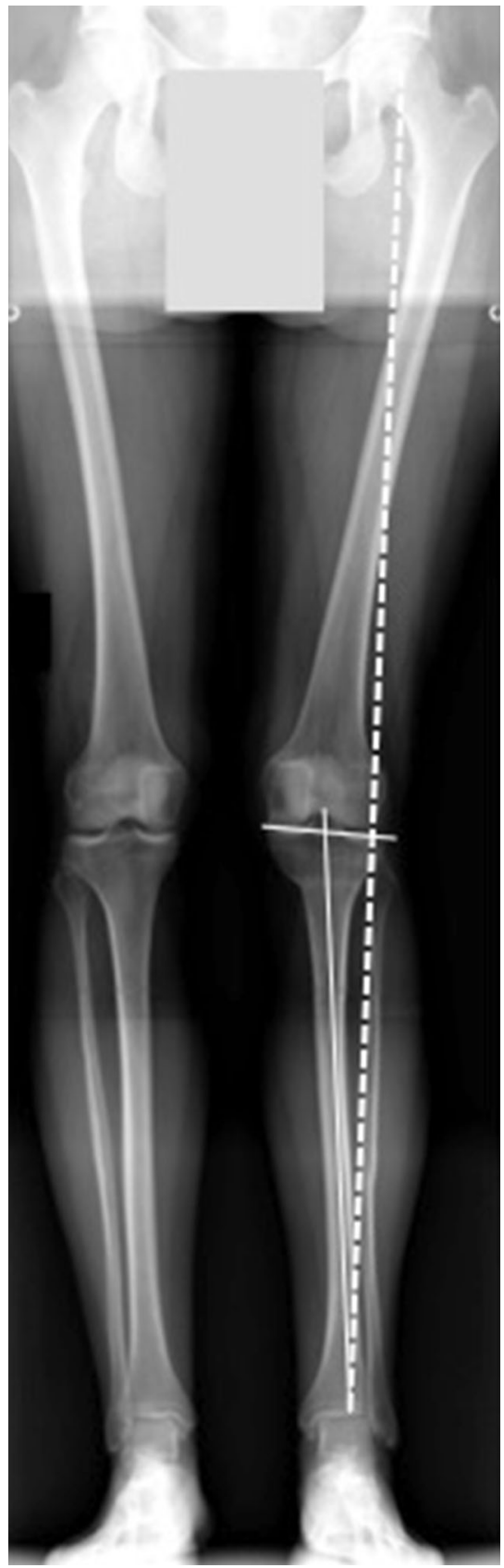

Abb. 1 \& Postoperative Ganzbeinaufnahme einer 48-jährigen Patientin nach proximaler tibialer Osteotomie (PTO) links mit Überkorrektur, bei der die Mickulicz-Linie weit lateral durch das laterale Gelenkkompartiment führt (gestrichelte Linie). Es kam ausserdem zu einer nach lateral abfallenden Gelenklinie, welche die allgemein akzeptierten $5^{\circ}$ überstieg (durchgehende Linien)

werden nicht berücksichtigt, genauso wie komplexe Achskorrekturen mit Fixateur, externe Techniken bzw. große extraartikuläre Korrekturen. Komplikationen, die auf einer fehlerhaften Planung beruhen, werden ebenfalls nicht in dieser Arbeit detailliert beschrieben. Für Informatio-

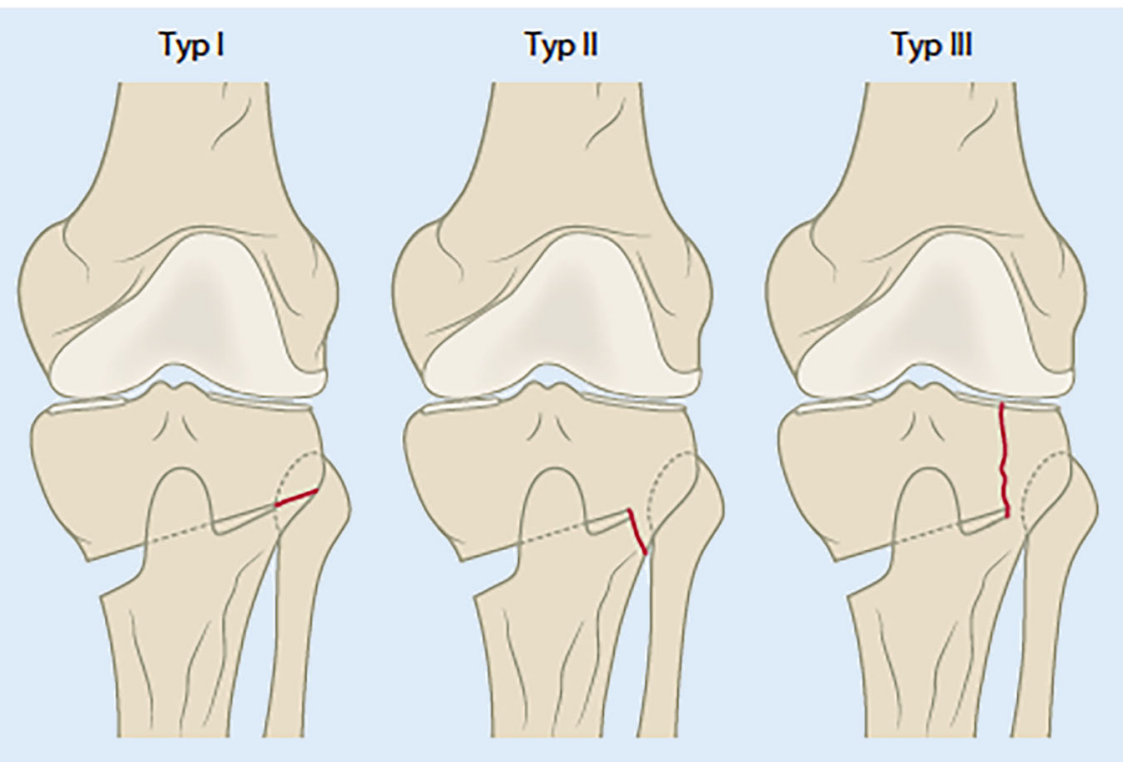

Abb. 2 A Scharnierfrakturen bei medial öffnender proximalen Tibiaosteotomie (PTO). Klassifikation nach Takeuchi et al. [39]: Typ I: auf Höhe des Tibiofibulargelenks, Typ II: unterhalb des Tibiofibulargelenks, Typ III: laterale Tibiaplateaufraktur. (Aus [30] mit freundl. Genehmigung)

nen über die herausragende Bedeutung der Planung für die Indikationsstellung und Durchführung von Beinachsenkorrekturen soll an dieser Stelle auf frühere Arbeiten verwiesen werden [14].

\section{Operative Lernkurve}

Einer der mitverantwortlichen Faktoren für die Komplikationsrate ist die Erfahrung des Operateurs. Da dieser nur schwer zu quantifizieren ist, wird er nur selten in der Literatur angegeben. Eine der wenigen Publikationen, die dies berücksichtigt hat, wurde 2010 von Chae et al. veröffentlicht [6]. Die Autoren begannen im Jahr 2003 mit der Durchführung von Umstellungsosteotomien, und im ersten Jahr erreichten sie eine eigentlich inakzeptable Komplikationsrate von $60 \%$. Im zweiten Jahr wurde sie um fast die Hälfte reduziert (32\%), und in den darauffolgenden Jahren sank sie weiter (2006: $23,3 \%$; 2007: 18,7\%), bis sie 2008 unter die akzeptable $5 \%$-Marke fiel. Dies verdeutlicht die Bedeutung der chirurgischen Erfahrung auf diesem Gebiet, was bei einer allgemein zurückgehenden Ausbildungszeit in der operativen Orthopädie und Traumatologie in Europa Fragen in Bezug auf die Qualität der Versorgung der Gesamtbevölkerung aufwerfen kann [33].
Dass dieser Faktor auch zu Fehlinterpretationen von wissenschaftlich hochrangigen Studien beitragen kann, konnte vor Kurzem anhand der Ergebnisse einer prospektiv-randomisierten Studie verdeutlicht werden [23]. Hier wurden die Ergebnisse der proximalen Tibiaosteotomie (PTO) in lateral geschlossener mit der medial öffnenden Technik verglichen. Weder in Bezug auf das klinische Ergebnis noch auf die Komplikationsrate konnte ein Unterschied zwischen den beiden Verfahrenstypen festgestellt werden. Allerdings lag die Komplikationsrate in beiden Gruppen bei außergewöhnlich hohen $33 \%$. Die Tatsache, dass die Ursache hierfür nicht diskutiert wurde, relativiert deswegen das Gesamtergebnis der Studie. Es kann demnach angenommen werden, dass die Erfahrung der Operateure das Ergebnis beeinflusst hat [32].

Diese Angaben verdeutlichen, dass Operateure, die während ihrer Facharztausbildung keine profunde Ausbildung im Bereich der kniegelenknahen Osteotomien genossen haben, sich das nötige Fachwissen in theoretischen und praktischen Kursen aneignen sollten, bevor sie mit diesen technisch komplexen und trotz der rezenten Fortschritte dennoch komplikationsanfälligen Eingriffen beginnen. 


\section{Komplikationsarten}

Teitge und Van Heerwaarden [40] haben die möglichen Komplikationen nach kniegelenknahen USO aufgelistet (• Tab. 2). In der vorliegenden Arbeit werden sie im Einzelnen ohne Anspruch auf Vollständigkeit aufgeführt und diskutiert. Die Komplikationen von Beinachsenkorrekturen können im Wesentlichen in 3 Kategorien unterteilt werden:

1. Komplikationen, die im Zusammenhang mit der Operationstechnik stehen,

2. biomechanische Komplikationen,

3. biologische Komplikationen.

\section{Biomechanische und technik- bedingte Komplikationen}

\section{Über-/Unterkorrektur}

Überkorrekturen entstehen am häufigsten nach proximalen tibialen Osteotomien (PTO), bei denen ein zu großer Korrekturwinkel unter Nichtberücksichtigung der Gelenklinie in der Frontalebene angestrebt wurde (•Abb. 1). Bei diesen Fällen kann entweder eine erneute Korrekturosteotomie durchgeführt werden, um das Ausmaß der Fehlstellung zu verringern, oder eine frühzeitige Konversion in eine Totalendoprothese vorgenommen werden. Größere Fallserien, die sich mit den Ergebnissen von fehlgeschlagenen Osteotomien beschäftigen oder die hervorheben, welche Vorgehensweise zu bevorzugen ist, wurden u. E. nach noch nicht veröffentlicht. Bei Unterkorrekturen oder Korrekturverlust der initialen Osteotomie kann eine Revisionsosteotomie zielführend sein. Alternativ kann die Implantation einer Schlittenprothese in Erwägung gezogen werden.

Eine andere Form der Überkorrektur stellt die Inklination der Gelenklinie in der Frontalebene dar. In der Regel fällt Letztere an der proximalen Tibia um $3^{\circ}$ nach medial ab. Wird die Korrekturosteotomie bei ausgeprägter Fehlstellung der Beinachse nur an einem Knochen durchgeführt, kann es zu einer unphysiologischen Inklination der Gelenklinie kommen. In den meisten Fällen ist dies bei Korrekturen von varischen Beinachsen und medial aufklappenden PTO der
Fall. Hierbei kommt es zu einer lateral abfallenden Gelenklinie, wodurch pathologische Scherkräfte im lateralen tibiofemoralen Kompartiment erzeugt werden. Dies kann bei den betroffenen Patienten trotz guter Korrektur der Gesamtbeinachse zu Schmerzen führen. Klinische und experimentelle Studien haben ergeben, dass der Gelenklinienwinkel $<5^{\circ}$ sein sollte [3, 22]. Seine Bestimmung sollte deswegen systematisch in die präoperative Planung aufgenommen werden.

\section{Scharnierfrakturen/Verlust der Primärstabilität}

Frakturen der Gegenkortikalis sollten vermieden werden, da sie zu Instabilitäten der Osteotomie führen können. Allerdings sind sie in den meisten Fällen mit den derzeit verbreiteten Operationstechniken auch bei technisch korrekter Durchführung der Operation kaum zu vermeiden. So haben Pape et al. gezeigt, dass es ab einer Öffnung des Osteotomiespalts von $8^{\circ}$ in $75 \%$ der Fälle $\mathrm{zu}$ einer solchen Fraktur kommen kann [26]. Ob und ab wann sie als regelrechte Operationskomplikation registriert werden können, bleibt demnach in der Diskussion. Die meisten Erkenntnisse zu diesen Frakturen gibt es derzeit bei PTO. Takeuchi et al. unterschieden zwischen 3 Frakturtypen ([39]; - Abb. 2). Neueste CT-basierte Untersuchungen haben ergeben, dass ihre Prävalenz zwischen 13,8 und $27,5 \%$ liegt $([7,13,16,20$, 37]; - Tab. 3). In vielen Fällen können sie bereits intraoperativ mit Hilfe einer Bildwandlerkontrolle diagnostiziert werden. Es konnte aber auch gezeigt werden, dass sie in seltenen Fällen noch postoperativ auftreten können [19]. Am besten sind sie mittels CT zu diagnostizieren und zu klassifizieren. Scharnierfrakturen bei distalen Femurosteotomien wurden bislang weder klassifiziert, noch gibt es Daten aus großen Fallserien zu ihrer Prävalenz.

Typ-I-Frakturen nach Takeuchi betreffen die Gegenkortikalis des proximalen Tibiakopfes (• Abb. 2). Etwa zwei Drittel aller Scharnierfrakturen sind vom Typ I. Sie haben in der Regel einen benignen Verlauf und heilen folgenlos aus.
Abstract

R. Seil · C. Mouton P. Schuster · V. A. Raoulis - A. Hoffmann - D. Pape M. Ollivier

\section{Lösungen für häufige Komplikationen bei Beinachsenkorrekturen}

\section{Zusammenfassung}

In den letzten 10 bis 15 Jahren kam es zu einem vermehrten wissenschaftlichen Interesse an den gelenkerhaltenden Verfahren der Gonarthrosebehandlung, insbesondere in Europa und Asien. Dies ist v. a. auf die Verfeinerung bestehender und die Entwicklung neuer Operationsmethoden und Osteosynthesetechniken zurückzuführen. Gleichzeitig ist der Eingriff technisch anspruchsvoll und kann eine ganze Reihe an Komplikationen hervorrufen. Diese sind entweder biomechanischer oder biologischer Natur. Der vorliegende Beitrag soll einen kurzen Überblick über die möglichen Komplikationen und deren Vermeidung geben.

\section{Schlüsselwörter}

Kniegelenk - Gonarthrose · Osteotomie . Osteosynthese. Gelenkerhaltende Chirurgie

\section{Solutions for complications after knee osteotomies}

\section{Abstract}

Over the last 10-15 years joint-preserving procedures for the treatment of gonarthritis have gained in popularity both in Europe and Asia. This is particularly due to refinement of existing surgical methods and the development of innovative surgical technologies and osteosynthesis. At the same time these surgical procedures are technically demanding and hence prone to a large number of complications, which can be biomechanical or biological in nature. This narrative article provides an overview of these complications, their causes and ways to prevent them.

Keywords

Gonarthritis - Osteotomy - Knee joint ·

Osteosynthesis · Joint-preserving surgery 


\begin{tabular}{|c|c|c|c|c|c|c|}
\hline \multirow[t]{2}{*}{ Autor } & \multirow{2}{*}{$\begin{array}{l}\text { Zahl der } \\
\text { Osteotomien }\end{array}$} & \multirow[t]{2}{*}{ Bildgebung } & \multirow{2}{*}{$\begin{array}{l}\text { Anzahl der Frakturen der } \\
\text { Gegenkortikalis }(n, \%)\end{array}$} & \multicolumn{3}{|c|}{ Frakturtyp nach Takeuchi (n, \% } \\
\hline & & & & I & II & III \\
\hline Chaouche [7] & 100 & CT nach 4 Wochen & $25(25)$ & $18(18)$ & $3(3)$ & $3(3)$ \\
\hline Lee [19] & 51 & CT nach 2 Tagen & $14(27,5)$ & $11(21,5)$ & $2(3,9)$ & $1(1,9)$ \\
\hline Kim [16] & 164 & $\begin{array}{l}\text { CT präoperativ, } 6 \text { Wochen, } 3,6,12 \text { Monate } \\
\text { postoperativ }\end{array}$ & $37(22,6)$ & $16(9,8)$ & $21(12,8)$ & 0 \\
\hline Lee u. Lee [20] & 94 & Röntgen, postoperative CT & $23(24)$ & $14(14,9)$ & $3(18,1)$ & $6(6,4)$ \\
\hline
\end{tabular}

Verläuft die Fraktur der Gegenkortikalis nach distal (Typ II), kann es zu hochgradigen Instabilitäten der Osteotomie führen, was sich in Form einer schmerzhaften Pseudarthrose oder bestenfalls einer verzögerten Knochenheilung ausdrücken kann (• Abb. 2). Auch sie können intraoperativ gerne übersehen werden. Werden sie korrekt nachbehandelt, heilen sie ebenso wie die Typ-IFrakturen gewöhnlich folgenlos aus [16, 19]. In Ausnahmefällen kann die Entlastungsphase bei solchen Patienten über 6 Wochen hinausgehen. $\mathrm{Zu}$ besonderer Vorsicht wird bei Patienten geraten, bei denen eine Typ-II-Fraktur gemeinsam mit einem anderen Risikofaktor zur verzögerten Knochenheilung, wie z. B. Rauchen, vorliegt [31].

Typ-III-Frakturen verlaufen nach proximal aufsteigend in den lateralen Gelenkspalt (• Abb. 2 und 3). Sie können zu einer schnellen Arthroseprogression im betroffenen Gelenkspalt führen und eine frühzeitige Konversion zur Totalendoprothese erforderlich machen. Sollte es intraoperativ zu einer solchen Fraktur kommen, kann eine zusätzliche osteosynthetische Stabilisierung, z. B. mittels Schraubenosteosynthese, in Erwägung gezogen werden. Wird sie erst postoperativ diagnostiziert, sollte die Entlastungsphase verlängert und der Patient frühzeitig über die eventuelle Notwendigkeit einer Revisionsoperation aufgeklärt werden. Eine systematische Revisionsoperation ab Diagnosestellung kann bei der aktuellen Datenlage noch nicht empfohlen werden.

Ein erster Schritt zur Verminderung der Prävalenz von Frakturen der Gegenkortikalis bei medial öffnender proximaler Tibiaosteotomie besteht darin, die Höhe der Osteotomie in der Frontalebene richtig zu bestimmen und sie in der von Chae als Sicherheitszone ausgewiesene
Breite durchzuführen [6]. Als Faustregel gilt, mit dem zur Vorlage benutzten ersten Kirschner-Draht von der Konkavität der medialen Tibiametaphyse ausgehend schräg nach proximal und lateral das proximale Drittel des Fibulaköpfchens anzuzielen. Hierbei muss jedoch beachtet werden, dass die Höhe des Fibulaköpfchens nicht wie in den meisten Fällen etwa $1 \mathrm{~cm}$ unterhalb der tibialen Gelenklinie endet, sondern sehr variabel sein kann [30]. Die Auf- bzw. Zuklappung der Osteotomie sollte mit großer Vorsicht, langsam und progressiv erfolgen. Die Elastizität des jeweiligen Knochens muss vom Operateur hierbei individuell eingeschätzt werden. Bei mangelnder Flexibilität muss überprüft werden, ob der Sägeschnitt ausreichend ist. Bei medial öffnender PTO muss eine Osteotomie überbrückende Bandspannung durch das oberflächliche mediale Kollateralband ausgeschlossen werden. Öffnet oder schließt die Osteotomie sich zu leicht, könnte eine instabile Fraktur der Gegenkortikalis vorliegen. Intraoperativ sollte die Integrität der Gegenkortikalis deswegen systematisch mittels Bildwandler überprüft werden. In solchen Fällen ist es von Vorteil, einen Plattenfixateur zu benutzen, der es ermöglicht, eine Kompression des Scharniers der Osteotomie zu erzeugen.

In den vergangenen Jahren wurden einige operationstechnische Variationen beschrieben, um das Scharnier der Osteotomie zu stärken und Frakturen der Gegenkortikalis zu vermeiden. Ein Ansatz bestand darin, ein in anteroposteriorer Richtung verlaufendes Bohrloch zur Entlastung der im Scharnierbereich auftretenden Biegekräfte anzubringen. Neueste biomechanische Untersuchungen haben ergeben, dass der Effekt dieser technischen Variante nur einen sehr geringen Vorteil bei kleinen Korrekturwin- keln bringt [28]. Eine andere Möglichkeit besteht darin, den lateralen Tibiakopf mit einem Kirschner-Draht temporär zu stabilisieren [8]. Abschließende Daten zur Effektivität dieser Methode liegen noch nicht vor.

》) Osteotomien mit Plattenfixateuren führen in aller Regel nach 6 Wochen zu einer knöchernen Ausheilung

Osteotomien mit Plattenfixateuren führen in aller Regel nach 6 Wochen $\mathrm{zu}$ einer knöchernen Ausheilung. Diese steht wiederum im direkten Zusammenhang mit der Präsenz einer Fraktur der Gegenkortikalis. So berichteten Schröter et al. über eine verminderte Frakturheilung beim Vorliegen einer Fraktur Typ II nach Takeuchi. Nach 6 Wochen konnte in $57 \pm 18 \%$ der Fälle mit TypII-Fraktur eine Ausheilung festgestellt werden, gegenüber $73 \pm 18 \%$ in Abwesenheit dieser Fraktur [30]. Ein Verlust der Primärstabilität der Osteosynthese führt dabei häufig zu Schmerzen bei Belastung, die über die 6-wöchigen Entlastungsphase hinausgehen. Bei $38 \%$ der Patienten nach proximaler, medial öffnender Tibiaosteotomie mittels TomoFix-Plattenfixateur (Depuy-Synthes, Oberdorf, Schweiz) konnte dies im normalen Verlauf festgestellt werden [27]. Bei ausgeprägten und nichtrückläufigen Beschwerden sollte dies Anlass $\mathrm{zu}$ einer weiteren diagnostischen $\mathrm{Ab}$ klärung sein. Röntgenologisch sollte bei Korrekturverlust oder dem unveränderten Bestehen oder sogar eine nach lateral verlaufende Erweiterung des Osteotomiespalts (-Abb. 4) im Vergleich zur postoperativen Röntgenkontrolle eine CT-Untersuchung durchgeführt werden. 

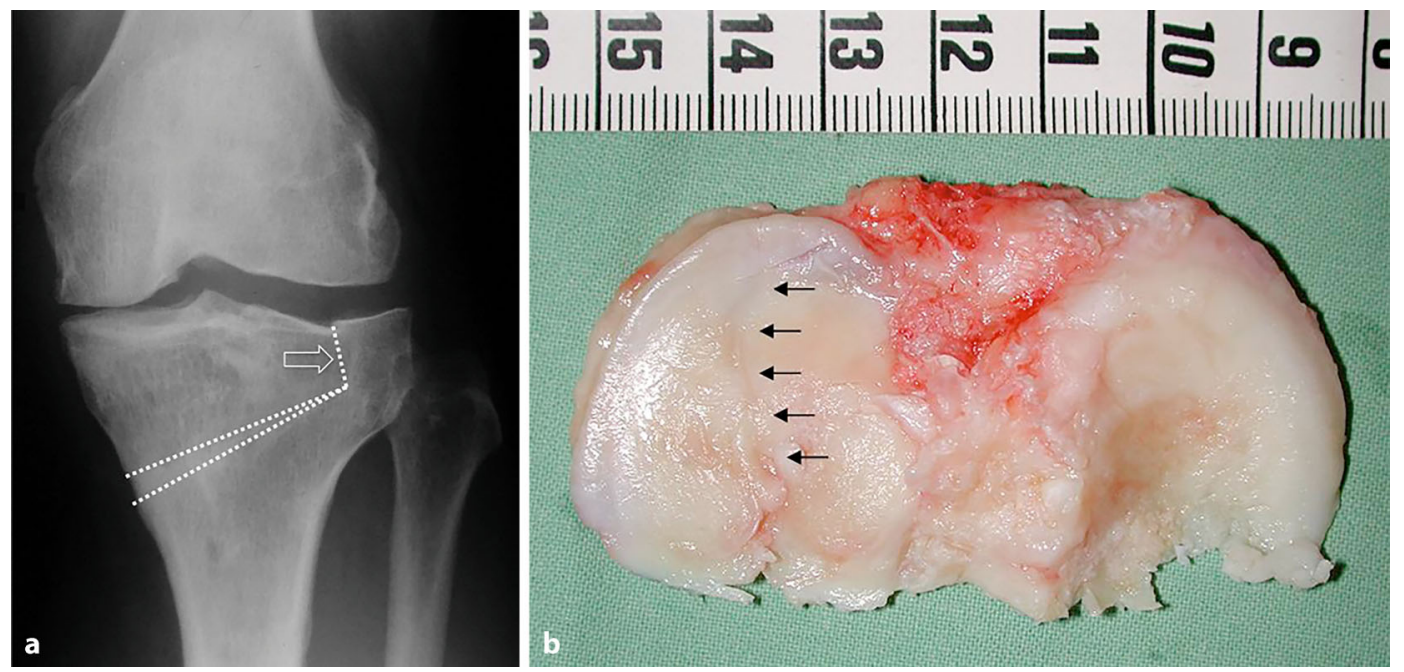

Abb. $3<$ a Postoperatives Röntgenbild einer nach proximal in den lateralen Gelenkspalt aufsteigende Typ-III-Fraktur nach Takeuchi (Pfeil) nach medial öffnender proximaler Tibiaosteotomie (PTO) bei einer 44-jährigen Patientin. b Reseziertes Tibiaplateau bei Konversion zur Knietotalendoprothese 5 Jahre nach der PTO. Die Pfeile stellen die noch gut sichtbare Frakturlinie durch das laterale Tibiaplateau dar

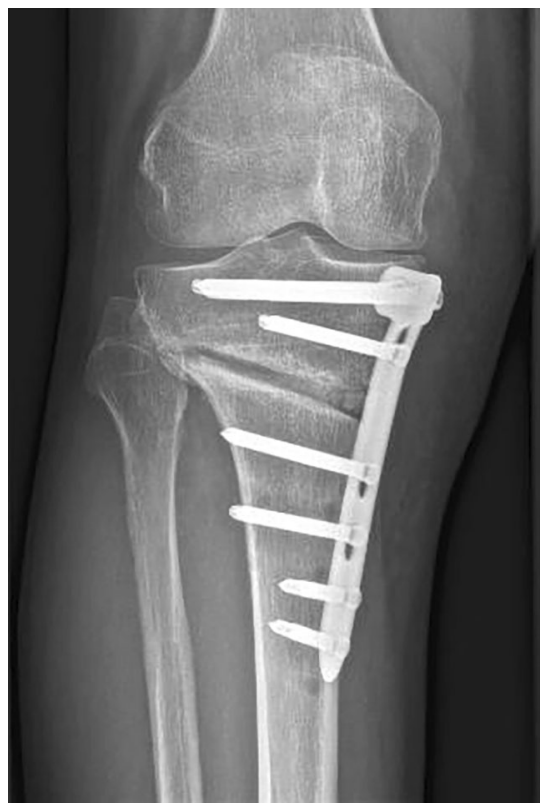

Abb. $4 \Delta$ Eine nach lateral verlaufende Erweiterung des Osteotomiespalts beim 6-Wochen-Follow-up sollte Anlass zu einer CT-Kontrolle sein, um z. B. eine instabile Fraktur vom Grad II nach Takeuchi auszuschließen

Moderne CT-Verfahren ermöglichen, Metallartefakte auf ein Minimum zu beschränken und ein verlässliches Urteil über mögliche Ursachen des Problems abzugeben. Hier können z.B. vorher nicht festgestellte Scharnierfrakturen ausgeschlossen werden, was die Autoren dazu veranlasste, eine systematische postoperative CT-Abklärung zu empfehlen, um bei instabilitätsgefährdeten Frakturen eine vorsichtigere Nachbehandlung durchführen zu können [20].

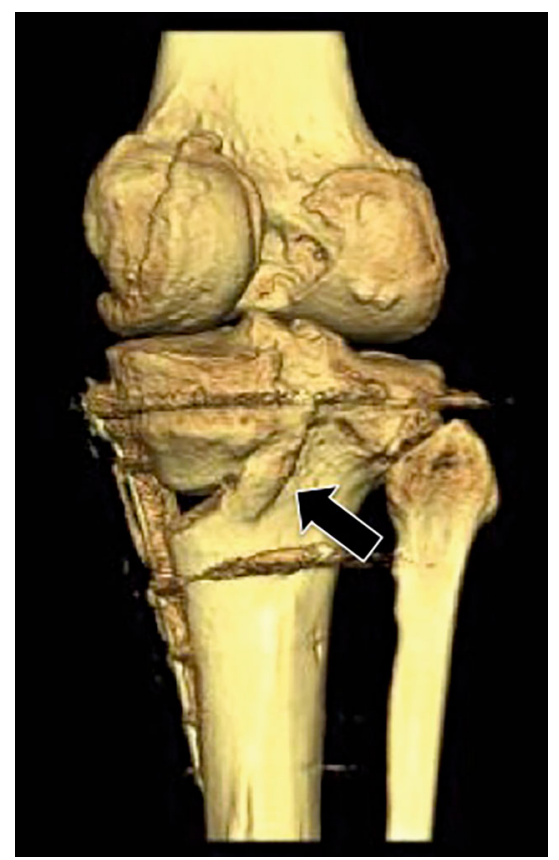

Abb. $5 \Delta$ Die Präsenz einer posteromedialen Kallusbildung bei CT-Untersuchungen nach 6 oder mehr Wochen stellt einen guten prognostischen Faktor dar (Pfeil)

Die Präsenz einer posteromedialen Kallusbildung bei CT-Untersuchungen nach 6 oder mehr Wochen stellt einen guten prognostischen Faktor dar (• Abb. 5). Röntgenologisch sollte die fachgerechte Durchführung der Operationstechnik kritisch beleuchtet werden. Oft kann diese einfache Untersuchung bereits zielführend sein. So kann beispielsweise geklärt werden, ob bei Benutzen des TomoFix-Fixateursystems eine Zugschraube verwendet wurde, um das laterale Scharnier zu komprimieren, denn das temporär besetzte Schraubenloch der Zugschraube kann noch lange nach der Osteotomie eingesehen werden. Auch kann festgestellt werden ob die Plattenhöhe nicht zu tief angelegt wurde, was zur Überbrückung des Osteotomiespalts mit den proximalen Schrauben des Plattenfixateurs und einer verzögerten Knochenheilung führen kann.

Implantatversagen sind mit den modernen Plattenfixateuren sehr selten geworden. Treten sie dennoch auf, so sind sie häufig Folge einer mangelnden technischen Ausführung der Operation. Ein klassisches Beispiel ist das fehlende Besetzen des "D-Lochs" des TomoFix-Systems, welches zu einer zu hohen Belastungskonzentration der Platte in diesem Bereich führt und ultimativ einen Plattenbruch erzeugen kann ( $\bullet$ Abb. 6). Neben Plattenbrüchen können auch Schraubenbrüche Anlass für symptomatische postoperative Verläufe sein. Lee et al. [21] berichteten über eine intraartikuläre Schraubendislokation nach medial zuklappender distaler Femurosteotomie. Ein klinisch oder röntgenologisch festgestellter Korrekturverlust sollte ebenfalls Anlass sein, ein Implantatversagen auszuschließen. In diesen Fällen muss eine Revisionsoperation mittels Reosteosynthese und Knochentransplantation erfolgen. In Abhängigkeit des Ausmaßes der knöchernen Dislokation kann eine Doppelosteosynthese in Erwägung gezogen werden (-Abb. 7). 


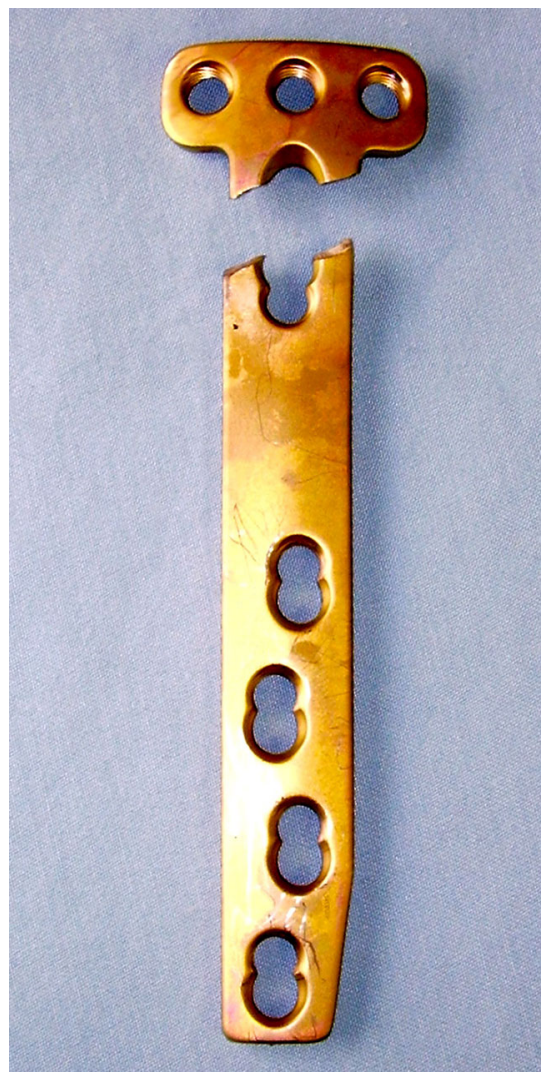

Abb. $6 \Delta$ Bruch eines Plattenfixateurs (TomoFix, Depuy-Synthes) durch fehlendes Besetzen des "D-Lochs". Dies hat zu einer zu hohen Belastungskonzentration der Platte in diesem Bereich geführt und ultimativ den Plattenbruch erzeugt

\section{Streckdefizit}

Bei proximaler Tibiaosteotomie kann ein iatrogenes Streckdefizit dann entstehen, wenn der Winkel zwischen der horizontalen und der aufsteigenden Osteotomie bei biplanarer Osteotomie zu flach gewählt oder intraoperativ im Moment der Osteosynthese nicht auf das Erreichen der vollen Streckstellung geachtet wird. Gleiches kann bei distaler Femurosteotomie entstehen. Auch sollte bei der medial öffnenden proximalen Tibiaosteotomie mittels biplanarer Technik darauf geachtet werden, die Dorsalneigung des Tibiaplateaus („tibial slope“) neutral zu halten. Hierbei sollte der Osteotomiespreizer im Moment der Aufklappung der Osteotomie an der dorsomedialen Tibiakante angebracht werden. Eine ventrale Platzierung des Spreizers führt zu einer Erhöhung der Neigung des Tibiaplateaus.

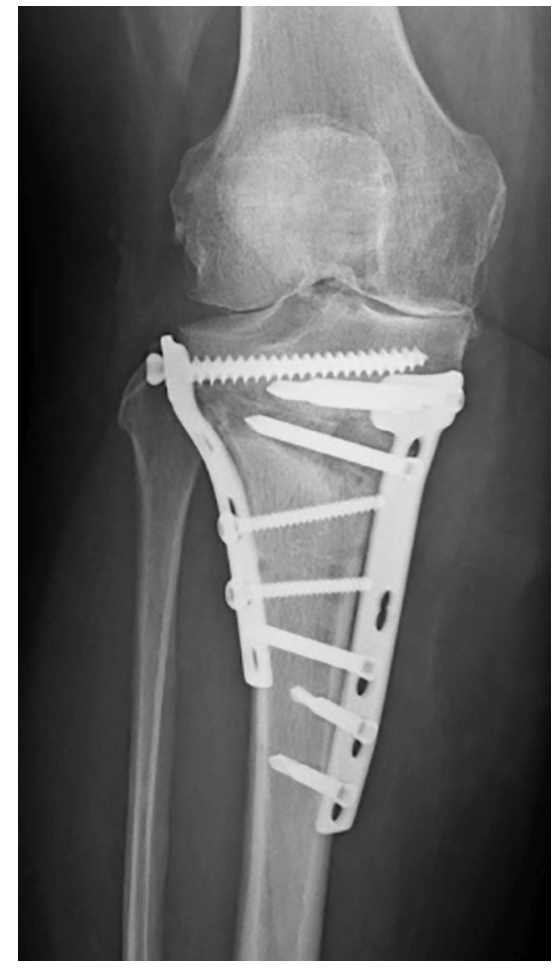

Abb. 7 \ Doppelosteosynthese und autologe Knochenspanplastik bei Patientin mit Plattenbruch

\section{Neurovaskuläre Komplikationen}

Wootton et al. [42] berichteten über $20 \%$ Schäden der Peronealnerven nach lateral schließender PTO. Die Autoren haben die Fibula in 4 Zonen unterteilt:

- Zone 1 entspricht dem Fibulakopf,

- Zone 2 beginnt am unteren Ende des Fibulakopfes und verläuft $7 \mathrm{~cm}$ nach distal

- Zone 3 betrifft die nächsten $8-15 \mathrm{~cm}$,

- Zone 4 betrifft den distalen Anteil der Fibula.

Das höchste Risiko, einen intraoperativen Nervenschaden zu erzeugen, besteht in den Zonen 2 und 3. Aydoglu et al. [2] haben die Fibula in 3 identische Drittel unterteilt und fanden das geringste Risiko am Übergang des mittleren zum distalen Drittel. Im Fall einer Fibulaosteotomie sollte insbesondere eine zu aggressive Elektrokoagulation vermieden werden. Gerich et al. [12] haben eine Übersichtsarbeit zu den sehr seltenen Gefäßverletzungen nach proximaler Tibiaosteotomie veröffentlicht. In der Regel handelte es sich um Einzelfälle mit Verletzungen der A. poplitea oder Pseudoaneurysmen derselben. Interessant war der sehr unterschiedliche Moment der Diagnosestellung der Verletzung, welcher von unmittelbar intraoperativ bis 3 Jahre postoperativ beschrieben wurde. Bei persistierenden Schmerzen im postoperativen Verlauf sollte eine Abklärung der A. poplitea zum Ausschluss eines Pseudoaneurysmas in Erwägung gezogen werden. Von besonderer chirurgischer Bedeutung ist der Abgang der A. tibialis anterior, welcher normalerweise dorsal vom und auf Höhe des M. popliteus verläuft, bevor sie die Membrana interossea durchbricht. Bei einigen Patienten verläuft dieser Abgang weiter proximal als gewöhnlich, was dazu führen kann, dass die Arterie ventral des M. popliteus verläuft. In diesen Fällen ist sie beim Durchsägen der dorsalen Tibiakortikalis gefährdet. Sollte dies vorkommen und intraoperativ festgestellt werden, so kann die Osteotomie aufgeklappt werden und eine direkte Blutstillung erfolgen.

\section{Patellofemorale Beschwerden}

Das Patellofemoralgelenk wird durch eine kniegelenknahe Osteotomie dahingehend beeinflusst, dass der Verlauf der Kniescheibe sich sowohl in der sagittalen Ebene als auch durch Rotationsveränderungen um wenige Millimeter verschieben kann $[10,18]$. Dies kann sowohl bei medial öffnenden als auch bei lateral schließenden PTO entstehen. In der Regel wird dies funktionell kompensiert und führt nicht zu patellofemoralen Beschwerden. Sollten diese dennoch nach adäquater Rehabilitation und Aufbau der Oberschenkelmuskulatur auftreten, sollte ausgeschlossen werden, ob die Tibiakopfosteotomie nicht zu einer Patella infera geführt hat. Prinzipiell gilt, dass eine medial aufklappende PTO die Patellahöhe vermindert, während eine lateral schließende PTO sie erhöht. Die iatrogene Patella infera kann vermieden werden, indem man die Osteotomie der Tuberositas tibiae in biplanarer Technik nach distal richtet [11]. In diesem Fall sollte die Tuberositas allerdings mit einer Schraubenosteosynthese verstärkt werden, um einen Ausriss durch einen zu starken Zug bei Quadrizepsaktivierung $\mathrm{zu}$ vermeiden. 


\section{Biologische Komplikationen}

Es konnte mehrfach nachgewiesen werden, dass Rauchen die Knochenheilung negativ beeinflusst [17, 35], weswegen die Indikation zur korrigierenden Umstellungsosteotomie bei Patienten, die das Rauchen nicht mehrere Monate vor dem Eingriff aufgeben können, kritisch überprüft werden sollte. Sie müssen zumindest auf die Möglichkeit der verzögerten Knochenheilung oder einer Pseudarthrose hingewiesen werden und eine verlängerte Entlastungsphase bzw. einen Zweiteingriff in Kauf nehmen.

Eine weitere relative Kontraindikation stellt das Übergewicht dar. Auch wenn noch keine klare Grenze gezogen werden konnte, ab welchem Body-Mass-Index (BMI) der Eingriff ggf. in Frage gestellt werden sollte, müssen adipöse Patienten ebenfalls auf die Möglichkeit einer erhöhten Komplikationsrate hingewiesen werden. Am ehesten ist bei diesen $\mathrm{Pa}$ tienten mit einer höheren Infektionsrate bzw. einer verzögerten Knochenheilung $\mathrm{zu}$ rechnen.

Infektionen nach kniegelenknahen Umstellungsosteotomien sind bei vorhandener Erfahrung mit diesen Eingriffen selten und liegen unter $1 \%$. Dennoch wurden in der Literatur sehr unterschiedliche Infektionsraten von $0,8-10,4 \%$ angegeben $[1,5,15,38,43]$. Handelt es sich um einen Spätinfekt, der nach abgeschlossener Knochenheilung auftritt, sollte die Osteotomieplatte entfernt, die Wunde gesäubert und eine keimgerechte Antibiotikatherapie angesetzt werden. Tritt der Infekt vor der knöchernen Ausheilung auf, sollte zumindest ein Versuch unternommen werden, den Infekt bei liegender Platte zu sanieren [4, 29]. Gelingt dies nicht, so muss eine alternative, temporäre Fixationsmethode mittels Fixateur externe oder Gipstutorimmobilisierung in Erwägung gezogen werden. Vor dieser Entscheidung empfehlen die Autoren eine systematische Abklärung der Knochenheilung mittels CT, um etwaige bereits bestehende Knochenbrücken aufzusuchen, wie dies beispielsweise im Bereich des lateralen Scharniers oder der Tuberositas tibiae bei medial aufsteigender proximaler Tibiaosteotomie der Fall sein kann.
Aufgrund der längeren Entlastungsoder Teilbelastungsphase muss nach der Korrekturosteotomie eine fachgerechte, mehrwöchige Thromboseprophylaxe durchgeführt werden.

Kutane Weichteilkomplikationen können nach vorangegangenen Hautvernarbungen auftreten. Wie bei anderen Eingriffen am Kniegelenk auch, muss dies in die präoperativen Überlegungen mit einbezogen werden. Um eine eventuell später anfallende Konversion in eine Knieprothese zu vereinfachen, empfehlen die Autoren, die Schnittführung weder bei der medial aufsteigenden noch bei der lateral schließenden proximalen Tibiaosteotomie schräg anzulegen. Hierbei kann es zu einer Konfluenz mit der längs verlaufenden Narbe der Knieprothese und Durchblutungsstörungen der Haut und Subkutis kommen.

Schließlich kann der venöse und lymphatische Rückfluss am Unterschenkel noch mehrere Monate nach kniegelenknaher Beinachsenkorrektur gestört sein und zu länger anhaltender Schwellneigung oder schlimmstenfalls zu einer Zellulitis oder einem Erysipel führen. Eine adäquate Kompressionsbehandlung und/oder Lymphdrainage kann hierbei wesentlich zur Besserung der Beschwerden beitragen.

\section{Fazit für die Praxis}

Kniegelenknahe Osteotomien zur
Beinachsenkorrektur in der Frontal-
ebene sind anspruchsvolle chirurgi-
sche Eingriffe, die eine Vielzahl an
Komplikationen hervorrufen können.
Werden sie operativ beherrscht, kann
die Zahl der intra- oder postope-
rativen Probleme allerdings unter
die 5\%-Rate gesenkt werden, was
mit Operationen eines ähnlichen
Schweregrads vergleichbar ist.
Die in diesem Beitrag beschriebenen
Tipps und Tricks können dabei helfen,
Komplikationen auf ein Minimum zu
reduzieren.

\section{Einhaltung ethischer Richtlinien}

Interessenkonflikt. R. Seil, C. Mouton, P. Schuster, V.A. Raoulis, A. Hoffmann, D. Pape und M. Ollivier geben an, dass kein Interessenkonflikt besteht.

Für diesen Beitrag wurden von den Autoren keine Studien an Menschen oder Tieren durchgeführt. Für die aufgeführten Studien gelten die jeweils dort angegebenen ethischen Richtlinien.

\section{Literatur}

1. Anagnostakos K, Mosser P, Kohn D (2013) Infections after high tibial osteotomy. Knee Surg Sports Traumatol Arthrosc 21:161-169

2. Aydogdu S, Yercan H, Saylam Cetal (1996) Peroneal nerve dysfunction after high tibial osteotomy. An anatomical cadaver study. Acta Orthop Belg 62:156-160

3. Babis GC, An KN, Chao EY et al (2002) Double level osteotomy of the knee: a method to retain jointline obliquity. Clinical results. J Bone Joint Surg Am $84: 1380-1388$

4. Berkes M, Obremskey WT, Scannell B et al (2010) Maintenance of hardware after early postoperative infection following fracture internal fixation. JBone Joint Surg Am 92:823-828

5. Bettin D, Karbowski A, Schwering L et al (1998) Time-dependent clinical and roentgenographical results of Coventry high tibial valgisation osteotomy. Arch Orthop Trauma Surg 117:53-57

6. Chae DJ, Shetty GM, Wang KH et al (2011) Early complications of medial opening wedge high tibial osteotomy using autologous tricortical iliac bone graft and T-plate fixation. Knee 18:278-284

7. Chaouche $S$, Jacquet $C$, Fabre-Aubrespy $M$ et al (2019) Patient-specific cutting guides for openwedge high tibial osteotomy: safety and accuracy analysis of a hundred patients continuous cohort. Int Orthop. https://doi.org/10.1007/s00264-01904372-4

8. Dessyn E, Sharma A, Donnez Met al (2019) Adding a protective K-wire during opening high tibial osteotomy increases lateral hinge resistance to fracture. Knee Surg Sports Traumatol Arthrosc https://doi.org/10.1007/s00167-019-05404-7

9. Floerkemeier S, Staubli AE, Schroeter S et al (2013) Outcome after high tibial open-wedge osteotomy: a retrospective evaluation of 533 patients. Knee Surg Sports Traumatol Arthrosc 21:170-180

10. Gaasbeek R, Welsing R, Barink M et al (2007) The influence of open and closed high tibial osteotomy on dynamic patellar tracking: a biomechanical study. Knee Surg Sports Traumatol Arthrosc 15:978-984

11. Gaasbeek RD, Sonneveld H, Van Heerwaarden RJ et al (2004) Distal tuberosity osteotomy in open wedge high tibial osteotomy can prevent patella infera: a new technique. Knee 11:457-461 
12. Gerich T, Lens V, Seil R et al (2014) Aufklappende Osteotomie des Tibiakopfes. Orthopäde 43:1008-1015

13. Han SB, Choi JH, Mahajan A et al (2019) Incidence and predictors of lateral hinge fractures following medial opening-wedge high tibial osteotomy using locking plate system: better performance of computed Tomography scans. J Arthroplasty 34:846-851

14. Holschen M, Lobenhoffer P (2016) Komplikationen kniegelenknaher Umstellungsosteotomien. Orthopäde 45:13-23

15. Ivarsson I, Myrnerts R, Gillquist J (1990) High tibial osteotomy for medial osteoarthritis of the knee. A 5 to 7 and 11 year follow-up. J Bone Joint Surg Br 72:238-244

16. Kim K-I, Kim GB, Kim HJ et al (2018) Extra-articular lateral hinge fracture does not affect the outcomes in medial open-wedge high tibial osteotomy using a locked plate system. Arthroscopy 34:3246-3255

17. Krannitz KW, Fong HW, Fallat LM et al (2009) The effect of cigarette smoking on radiographic bone healing after elective foot surgery. J Foot Ankle Surg 48:525-527

18. Kuwashima U, Takeuchi R, Ishikawa H et al (2019) Comparison of torsional changes in the tibia following a lateral closed or medial open wedge high tibial osteotomy. Knee 26:374-381

19. Lee BS, Jo BK, Bin SI et al (2019) Hinge fractures are underestimated on plain radiographs after open wedge proximal tibial osteotomy: evaluation by computed Tomography. Am J Sports Med 47:1370-1375

20. Lee OS, Lee YS (2018) Diagnostic value of computed Tomography and risk factors for lateral hinge fracture in the open wedge high tibial osteotomy. Arthroscopy 34:1032-1043

21. Lee YS, Lee SH, Lee ES et al (2019) Case report: migration of a broken screw to the knee joint after hardware failure following closing wedge distal femoral osteotomy. BMC Musculoskelet Disord 20:118

22. Nakayama H, Schroter S, Yamamoto C et al (2018) Large correction in opening wedge high tibial osteotomy with resultant joint-line obliquity induces excessive shear stress on the articular cartilage. Knee Surg Sports Traumatol Arthrosc 26:1873-1878

23. Nerhus TK, Ekeland A, Solberg G et al (2017) No difference in time-dependent improvement in functional outcome following closing wedge versus opening wedge high tibial osteotomy: a randomised controlled trial with two-year followup. Bone Joint J99-B:1157-1166

24. Niemeyer P, Koestler W, Kaehny C et al (2008) Twoyear results of open-wedge high tibial osteotomy with fixation by medial plate fixator for medial compartment arthritis with varus malalignment of the knee. Arthroscopy 24:796-804

25. Palmer H, Elson D, Baddeley T et al (2018) The United Kingdom Knee Osteotomy Registry: The First Annual Report 2018. https://secure. amplitude-registry.com/UKKOR/Content/Ukkor AnnualReport_RGB.pdf.Zugegriffen: 15.8.2019

26. Pape D, Adam F, Seil R et al (2005) Fixation stability following high tibial osteotomy: a radiostereometric analysis. J Knee Surg 18:108-115

27. Pape D, Dueck K, Haag M et al (2013) Wedge volume and osteotomy surface depend on surgical technique for high tibial osteotomy. Knee Surg Sports Traumatol Arthrosc 21:127-133

28. Reyle G, Lorbach O, Diffo Kaze A et al (2017) Verhinderung einer Fraktur der Gegenkortika- lis bei aufklappender Osteotomie. Orthopäde 46:610-616

29. Rightmire E, Zurakowski D, Vrahas M (2008) Acute infections after fracture repair: management with hardware in place. Clin Orthop Relat Res 466:466-472

30. Schröter S, Ateschrang A, Ihle C et al (2014) Die Fraktur der Gegenkortikalis bei der aufklappenden Osteotomie des Tibiakopfes. Orthopäde 43:1000-1007

31. Schroter S, Freude T, Kopp MMet al (2015) Smoking and unstable hinge fractures cause delayed gap filling irrespective of early weight bearing after open wedge osteotomy. Arthroscopy 31:254-265

32. Seil R (2018) In high tibial osteotomy, closing and opening wedges did not differ for clinical outcomes at up to two years. J Bone Joint Surg 100:882

33. Seil R (2019) Why ESSKA? On what it takes for orthopaedic surgeons and their scientific societies to adapt to societal changes in 2018. Knee Surg Sports Traumatol Arthrosc 27:665-672

34. Seil R, Van Heerwaarden $R$, Lobenhoffer $P$ et al (2013) The rapid evolution of knee osteotomies. Knee Surg Sports Traumatol Arthrosc 21:1-2

35. Sloan A, Hussain I, Maqsood M et al (2010) The effects of smoking on fracture healing. Surgeon 8:111-116

36. Song EK, Seon JK, Park SJ et al (2010) The complications of high tibial osteotomy: closingversus opening-wedge methods. J Bone Joint Surg Br92:1245-1252

37. Song KY, Koh IJ, Kim MS et al (2019) Early experience of lateral hinge fracture during medial opening-wedge high tibial osteotomy: incidence and clinical outcomes. Arch Orthop Trauma Surg. https://doi.org/10.1007/s00402-019-03237-0

38. Sprenger TR, Doerzbacher JF (2003) Tibial osteotomy for the treatment of varus gonarthrosis. Survival and failure analysis to twenty-two years. JBone Joint Surg Am 85:469-474

39. Takeuchi R, Ishikawa H, Kumagai K et al (2012) Fractures around the lateral cortical hinge after a medial opening-wedge high tibial osteotomy: a new classification of lateral hinge fracture. Arthroscopy 28:85-94

40. Teitge RA, Van Heerwaarden RJ (2013) 18 Osteotomies for failed osteotomies around the knee. In: Lobenhoffer PP, van Heerwaarden RJ, Staubli AE, Jakob RP, Galla M, Agneskirchner JD (Hrsg) Osteotomies around the Knee. Thieme, Stuttgart

41. Van Den Bekerom MP, Patt TW, Kleinhout MY et al (2008) Early complications after high tibial osteotomy: a comparison of two techniques.JKnee Surg 21:68-74

42. Wootton JR, Ashworth MJ, Maclaren CA (1995) Neurological complications of high tibial osteoto my - the fibular osteotomy as a causative factor: a clinical and anatomical study. Ann R Coll Surg Engl 77:31-34

43. Wu LD, Hahne HJ, Hassenpflug T (2004) A longterm follow-up study of high tibial osteotomy for medial compartment osteoarthrosis. Chin J Traumatol 7:348-353 